\title{
Thiol-Disulfide Homeostasis in Patients with Panic Disorder
}

\author{
Burak Kulaksizoglu1, Sibel Kulaksizoglu ${ }^{2 *}$ \\ ${ }^{1}$ Psychiatry Department, Antalya Education and Research Hospital, Antalya, Turkey \\ ${ }^{2}$ Biochemistry Department, Antalya Education and Research Hospital, Antalya, Turkey \\ Email: *sblclngrgl@yahoo.co.uk
}

How to cite this paper: Kulaksizoglu, B. and Kulaksizoglu, S. (2017) Thiol-Disulfide Homeostasis in Patients with Panic Disorder. International Journal of Clinical Medicine, 8, 34-41.

http://dx.doi.org/10.4236/ijcm.2017.81004

Received: December 20, 2016

Accepted: January 19, 2017

Published: January 22, 2017

Copyright ( 92017 by authors and Scientific Research Publishing Inc. This work is licensed under the Creative Commons Attribution International License (CC BY 4.0).

http://creativecommons.org/licenses/by/4.0/

\section{c) (i) Open Access}

\begin{abstract}
Objective: To determine serum thiol/disulfide homeostasis in panic disorder (PD). Methods: Serum native thiol, total thiol, and disulfide levels were measured in the patients with $40 \mathrm{PD}$ patients and 40 healthy subjects. Serum native thiol, total thiol, and disulfide levels were measured with a novel colorimetric, automated method. The thiol-disulfide ratio was also calculated. Results: The native thiol $(\mathrm{p}<0.001)$ and total thiol $(\mathrm{p}<0.001)$ levels, and the native thiol/total thiol $(\mathrm{p}<0.001)$ ratio were significantly lower, whereas disulfide/native thiol $(\mathrm{p}<0.001)$ and disulfide/total thiol $(\mathrm{p}<0.001)$ ratio significantly increased in the PD patient group compared to the control group. The cut-off value was $92.26,3.83$ and 3.56 for native thiol/total thiol, disulfide/native thiol and disulfide/total thiol respectively. Conclusion: This is the first study in the literature to evaluate thiol-disulfide homeostasis in patients with PD. Our results suggest that the disulfide/thiol ratio is significantly greater in panic disorder patients.
\end{abstract}

\section{Keywords}

Panic Disorder, Thiol-Disulphide Homeostasis

\section{Introduction}

Despite significant progress in studies on PD, etiopathogenic processes cannot be clearly defined. One of the important processes in the pathogenesis of PD is oxidative stress [1]. Oxidative stress can be described as the impairment of cell membrane functions due to the disequilibrium between the free oxygen radicals and the antioxidant mechanisms. Oxidative stress can be evaluated indirectly by the measurement of some antioxidant enzyme levels such as superoxide dismutase (SOD), catalase (CAT), or glutathione peroxidase (GSH-Px), by-products of lipid peroxidation such as malondialdehyde (MDA) [2]. Although many impor- 
tant findings have been revealed by studies on oxidative and anti-oxidative parameters (MDA, SOD, glutathione (GSH), adenosine deaminase (ADA), xanthine oxidase (XO), total antioxidant status (TAS), total oxidative status (TOS), oxidative stress index (OSI), ceruloplasmin) related to PD etiology, oxidant-antioxidant homeostasis of PD is not fully clear [1] [3] [4].

The thiol groups are the primary targets of the reactive oxygen species. Thiol is an organic compound containing sulfhydryl $(-\mathrm{SH})$ group which has a critical role in preventing the occurrence of oxidative stress. Thiol groups are oxidized by reactive oxygen species and reversible disulfide bonds are formed. This is the earliest sign of protein oxidation [5] [6] [7]. The most of thiols in plasma are formed by albumin, and the remaining part of the thiols consists of low molecular weighted thiols such as homocysteine, methionine, cysteine, cysteinyl glycine, glutathione, and $\gamma$-glutamylcysteine [8]. Thiol groups of proteins are oxidized by oxidant molecules and reversibly converted to disulfide bond structure. Disulfide bond structure can be again reduced to the thiol group. Maintenance of the thiol-disulfide homeostasis is kept by conversion of disulfide bond structure to the thiol group. When the native thiol levels decrease, disulfide levels increase [9].

Thiol-disulfide homeostasis has an important role on antioxidant protection, detoxification, apoptosis, stabilization of protein structure, regulation of protein function, cell signaling and transcription [6]. While only the thiol levels were able to be measured since 1979, a novel and colorimetric, automated method was developed by Erel and Neselioglu so that the thiol and also the disulfide levels can be measured one by one [9] [10]. Abnormal thiol-disulfide homeostasis state is related to the pathogenesis of different diseases, including diabetes mellitus [11], cardiovascular diseases [12], cancers [13], neurological diseases [14] [15], liver diseases [16] and chronic renal failure [17].

There is no study in the literature showing the relationship between PD with the thiol-disulfide homeostasis. The aim of our study is to investigate the thioldisulfide homeostasis in patients with PD.

\section{Material and Method}

Totally 80 volunteers; 40 ( 15 males, 25 females) PD patients and 40 (20 males, 20 females) healthy controls aged between 18 - 65 years old, were participated into study. Informed consent was obtained from the patient and control groups. The PD patients were enrolled at the first admission, to the psychiatry clinic of Antalya Education and Research Hospital, between July 2016 and October 2016, and had no treatment, were diagnosed according to the diagnostic criteria of Diagnostic and Statistical Manual of Mental Disorders, Fourth Edition, Text Revision (DSM-IV-TR) [18] Control group was recruited from healthy persons who were hospital staff and were assessed by a semi structured psychiatric interview. The study complied with the Declaration of Helsinki, and was approved by institutional ethics committee of Antalya Training and Research Hospital.

Patients were excluded from the study if they met one or more of the follow- 
ing criteria: hypertension, heart and cerebrovascular disease, diabetes mellitus, hepatic or renal failure, autoimmune diseases, active infection, active or chronic inflammatory diseases, smoking, obesity, collagen tissue disease and treatment with antiinflammatory, antioxidant or immunosuppressive medications, malignity, alcohol and substance abuse or dependence, vitamin supplements. Sociodemographic data form was completed to the participants.

Antecubital vein blood was taken after 12 hours of fasting from the participant. Vacutainer gel tubes were used. Serum was separated by centrifugation 10 min at $4000 \mathrm{rpm}$, at $4^{\circ} \mathrm{C}$ and rapidly serum fractions were stored at $-80^{\circ} \mathrm{C}$.

A new and fully colorimetric automated method developed by Erel et al. was used for the measurement of plasma native thiol, total thiol and disulfide levels based on the reduction of dynamic disulfide bonds to functional thiol groups. Native thiol content was subtracted from the total thiol content; half of the obtained difference provides the disulfide bond quantity. Disulfide/thiol ratio, disulfide/total thiol ratio and thiol/total thiol ratios were calculated automatically [9].

\section{Statistical Analysis}

In the statistical evaluation of the data, the statistics package for social sciences (SPSS) Version 18.0 computer package program was used. The results were presented as mean $\pm \mathrm{SD}$. The differences between groups were determined by student's unpaired $t$-test and Mann Whitney U-test. Pearson correlation coefficient was used to assess correlations. Distribution of continuous variables was assessed with Kolmogorov-Smirnoy. Receiver operating characteristic (ROC) curve analysis was used to determine the optimum cut-off levels of native thiol, total thiol, disulfide/native thiol and disulfide/total thiol. Using the ROC curve, the responsiveness is described in terms of sensitivity and specificity. Values for sensitivity and for false-positive rates (1-specificity) are plotted on the $y$-and the $x$-axes of the curve and the area under the curve represents the probability a measure correctly classifies patients as improved or unchanged. $\mathrm{P}$ values less than 0.05 was accepted as the significance level.

\section{Results}

The mean ages among the PD group (15 male, 37.5\%) were $39.9 \pm 14.4$ years, and the mean ages of the control group (20 male, 50\%) were $38.2 \pm 13.6$ years. The age and gender distributions were similar in both groups $(\mathrm{p}=0.594,0.260$, respectively).

The laboratory findings of the control and PD groups are in Table 1. Native and total thiol levels were significantly lower among the PD group relative to the control group ( $p<0.001)$. There was no significant difference between the control and the PD groups in terms of disulfide levels $(p=0.261)$. While the native thiol/total thiol ratio was decreased in the patient group $(\mathrm{p}<0.001)$, disulfide/ native thiol and disulfide/total thiol ratios were elevated in the patient group $(\mathrm{p}$ $<0.001, \mathrm{p}<0.001$ respectively). 
ROC analysis of area under the curve, cut-off levels, and sensitivity and specificity values are given in Table 2. The AUC for the Native Thiol and Native Thiol/Total Thiol ratio were 0.855 and 0.793 respectively.

ROC analysis was used. ROC curves of all parameters are seen in Figure 1. The sensitivity and specificity of Total Thiol were $80 \%$ and $87.5 \%$, respectively, and the AUC was 0.837 .

\section{Discussion}

The relationship of oxidative stress between PD was demonstrated in different studies. Several studies conducted in patients with PD related to oxidative stress have evaluated molecules such as MDA, SOD, GSH, CAT, nitric oxide (NO), uric acid, and paraoxonase (PON); however, more recent studies have also evaluated distinct parameters, such as TAS, TOS, and OSI [1] [3] [4]. These studies have yielded variable results.

In a study done by Gul et al., the oxidant (MDA) was found higher and antioxidant (total antioxidant capacity (TAC), PON, arylesterase (ARE)) levels lower in PD patients compared to the control group [3]. Otherwise Kuloglu et al.,

Table 1. Comparison of the laboratory findings between the control group and PD group.

\begin{tabular}{cccc}
\hline & $\begin{array}{c}\text { Patient Group } \\
(\mathrm{n}=40) \\
\text { Mean } \pm \text { SD }\end{array}$ & $\begin{array}{c}\text { Control Group } \\
(\mathrm{n}=40) \\
\text { Mean } \pm \text { SD }\end{array}$ & $\mathrm{p}$ \\
\hline Native Thiol $(\mu \mathrm{mol} / \mathrm{l})$ & $302.90 \pm 90.95$ & $413.66 \pm 46.27$ & $<0.001^{*}$ \\
Total Thiol $(\mu \mathrm{mol} / \mathrm{l})$ & $333.70 \pm 92.18$ & $442.09 \pm 48.63$ & $<0.001^{*}$ \\
Disulfide $(\mu \mathrm{mol} / \mathrm{l})$ & $15.40 \pm 3.23$ & $14.22 \pm 5.77$ & 0.261 \\
Disulfide/Native Thiol (\%) & $5.67 \pm 2.63$ & $3.47 \pm 1.46$ & $<0.001^{*}$ \\
Disulfide/Total Thiol (\%) & $5.00 \pm 1.96$ & $3.22 \pm 1.26$ & $<0.001^{*}$ \\
Native Thiol/Total Thiol $(\%)$ & $90.00 \pm 3.92$ & $93.57 \pm 2.53$ & $<0.001^{*}$
\end{tabular}

${ }^{\star}$ Correlation is significant at the 0.05 level (2-tailed). Students $t$ test was used.

Table 2. ROC analysis data of Native Thiol, Total Thiol, Disulfide, Disulfide/Native Thiol, Disulfide/Total Thiol, Native Thiol/ Total Thiol parameters.

\begin{tabular}{cccccccc}
\hline Biomarker & $\begin{array}{c}\text { Area Under Curve } \\
\text { (AUC) }\end{array}$ & P value & Cut-off level & Sensitivity (\%) & Specificity (\%) & + LR & - LR \\
\hline Native Thiol & 0.855 & $<0.001$ & 353.1 & 75.00 & 92.50 & 10.0 & 0.27 \\
Total Thiol & 0.837 & $<0.001$ & 393.8 & 80.00 & 87.50 & 6.40 & 0.23 \\
Disulfide & 0.579 & 0.233 & 10.6 & 97.50 & 32.50 & 1.44 & 0.07 \\
Disulfide/Native Thiol & 0.793 & $<0.001$ & 3.83 & 82.50 & 65.00 & 2.36 & 0.27 \\
Disulfide/Total Thiol & 0.793 & $<0.001$ & 3.56 & 82.50 & 65.00 & 2.36 & 0.27 \\
Native Thiol/Total Thiol & 0.793 & $<0.001$ & 92.26 & 77.50 & 70.00 & 2.58 & 0.32 \\
\hline
\end{tabular}

$+\mathrm{LR}=$ Positive likelihood ratio; $-\mathrm{LR}=$ Negative likelihood ratio. 


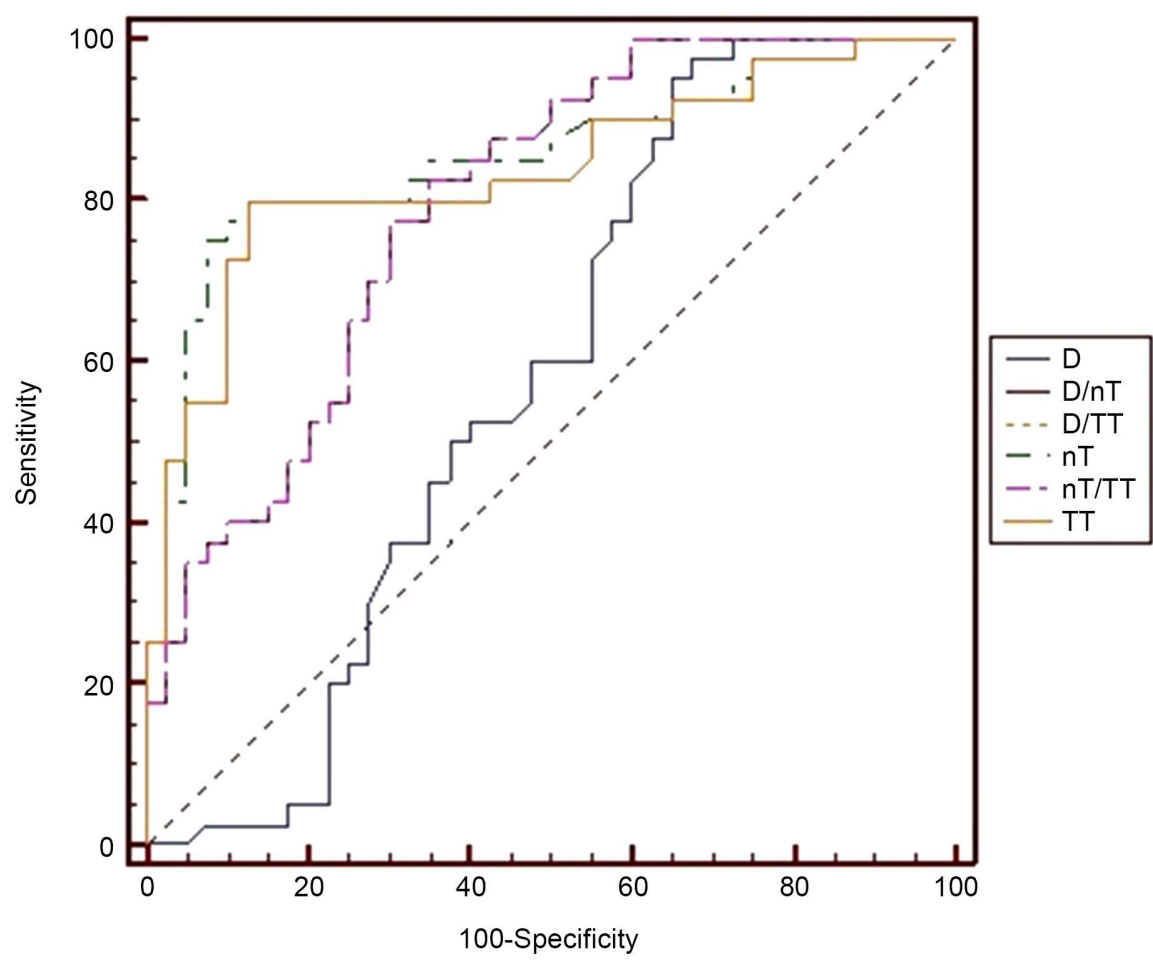

Figure 1. ROC analysis of PD patients versus controls.

found the GSH-Px, SOD and MDA levels to be significantly higher in the PD group compared to the control group [19]. Another study has again evaluated oxidative and anti-oxidative parameters in PD. Oxidants (adenosine deaminase (ADA) and XO levels) were higher in the patient group than the control group in this study but there was no significant difference regarding antioxidant parameters [20]. Although many important findings have been revealed by studies on oxidative and anti-oxidative PD etiology [19] [20], oxidant-antioxidant homeostasis of $\mathrm{PD}$ has not been fully elucidated, yet.

The thiol-disulfide homeostasis plays a role in the cellular functions. The thiols are the primary target for oxygen radicals which are non-enzymatic antioxidant molecules taking an important part in preventing damage caused by free radicals [21]. The extent to which the proteins are affected from free radicals is related to their amino acid content. Molecules containing unsaturated bonds and SH groups and proteins releasing amino acids such as homocysteine, methionine, cysteine, tryptophan, tyrosine, phenylalanine, and histidine can be more easily affected by free radicals [22] [23]. Thiol-disulfide homeostasis, which was first measured in 1979, could be only measured in one direction [10]; however, a novel, automated and colorimetric method developed in 2014 by Erel et al. which allows the measurement of the homeostasis in either direction. Erel et al. reported that disulfide levels were higher in patients with diseases such as diabetes, in smokers, obese patients, premature ovarian failure, subclinical hypothyroidism and were lower in patients with proliferative diseases, such as urinary bladder cancer, renal cancer, non-small cell lung cancer and multiple myeloma [9]. 
This is the first study in the literature to evaluate thiol-disulfide homeostasis in patients with PD. The present study found decreased levels of native thiol, total thiol, and native thiol/total thiol ratio and increased disulfide/native thiol and disulfide/total thiol levels in the patient group, compared to the control group. These finding suggest that thiol-disulfide homeostasis has shifted toward disulfide direction in PD patients. Decreased thiol levels indicate a problem in antioxidant system in PD patients. According to our data, the cut-off value was $353.1 \mu \mathrm{mol} / \mathrm{L}, 393.8 \mu \mathrm{mol} / \mathrm{L}, 92.26$ (\%), 3.83 and 3.56 for native thiol, total thiol, native thiol/total thiol, disulfide/native thiol and disulfide/total thiol level respectively. The patients with serum native thiol, total thiol and native thiol/ total thiol levels below these sills must be particularly evaluated for PD. The evaluation can be made by the automated measurement of these values.

Although the increase in disulfide molecules generated by oxidation of thiol groups in the patient group was not statistically significant, increased disulfide/ native thiol and disulfide/total thiol levels suggested increased oxidative stress in PD patients. The deficiency of a significant increase in disulfide levels in the PD group can be explained by the lack of stability in the thiol-disulfide homeostasis and the fact that this balance is affected by kidney and liver functions [24].

This study is cross sectional so we think that this is the main limitation. The sample size of the patient and control groups may be another limitation.

\section{Conclusion}

The present study investigated thiol-disulfide homeostasis using a new, colorimetric, and automated method in PD patients. Our study results showed that serum thiol levels were lower and disulfide/total thiol levels were higher in PD patients, compared to the controls. These findings also highlight the importance of oxidative stress in the etiopathogenesis of PD. We believe that further studies investigating the effects of $\mathrm{SH}$-containing anti-oxidant components on thioldisulfide homeostasis and clinical outcomes of PD treatment would make significant contribution to the treatment of this disease.

\section{References}

[1] Ersoy, M.A., Selek, S., Celik, H., Erel, O., Kaya, M.C., Savas, H.A., et al. (2008) Role of Oxidative and Antioxidative Parameters in Etiopathogenesis and Prognosis of Panic Disorder. International Journal of Neuroscience, 118, 1025-1037. https://doi.org/10.1080/00207450701769026

[2] Rahal, A., Kumar, A., Singh, V., Yadav, B., Tiwari, R., Chakraborty, S., et al. (2014) Oxidative Stress, Prooxidants, and Antioxidants: The Interplay. BioMed Research International, 2014, Article ID: 761264. https://doi.org/10.1155/2014/761264

[3] Gul, I.G., Karlidag, R., Cumurcu, B.E., Turkoz, Y., Kartalci, S., Ozcan, A.C., et al. (2013) The Effect of Agoraphobia on Oxidative Stress in Panic Disorder. Psychiatry Investigation, 10, 317-325. https://doi.org/10.4306/pi.2013.10.4.317

[4] Bouayed, J., Rammal, H. and Soulimani, R. (2009) Oxidative Stress and Anxiety: Relationship and Cellular Pathways. Oxidative Medicine and Cellular Longevity, 2, 63 67. https://doi.org/10.4161/oxim.2.2.7944 
[5] Cadenas, E. (1989) Biochemistry of Oxygen Toxicity. Annual Review of Biochemistry, 58, 79-110. https://doi.org/10.1146/annurev.bi.58.070189.000455

[6] Tetik, S., Ahmad, S., Alturfan, A.A., Fresko, I., Disbudak, M., Sahin, Y., et al. (2010) Determination of Oxidant Stress in Plasma of Rheumatoid Arthritis and Primary Osteoarthritis Patients. Indian Journal of Biochemistry \& Biophysics, 47, 353-358.

[7] Nagy, P. (2013) Kinetics and Mechanisms of Thiol-Disulfide Exchange Covering Direct Substitution and Thiol Oxidation-Mediated Pathways. Antioxidants \& Redox Signaling, 18, 1623-1641. https://doi.org/10.1089/ars.2012.4973

[8] Turell, L., Radi, R. and Alvarez, B. (2013) The Thiol Pool in Human Plasma: The Central Contribution of Albumin to Redox Processes. Free Radical Biology \& Medicine, 65, 244-253. https://doi.org/10.1016/j.freeradbiomed.2013.05.050

[9] Erel, O. and Neselioglu, S. (2014) A Novel and Automated Assay for Thiol/ Disulphide Homeostasis. Clinical Biochemistry, 47, 326-332. https://doi.org/10.1016/j.clinbiochem.2014.09.026

[10] Ellman, G. and Lysko, H. (1979) A Precise Method for the Determination of Plasma Sulfhydryl Groups. Analytical Biochemistry, 93, 98-102. https://doi.org/10.1016/S0003-2697(79)80122-0

[11] Matteucci, E. and Giampietro, O. (2010) Thiol Signalling Network with an Eye to Diabetes. Molecules, 15, 8890-8903. https://doi.org/10.3390/molecules15128890

[12] Go, Y.M. and Jones, D.P. (2011) Cysteine/Cystine Redox Signaling in Cardiovascular Disease. Free Radical Biology \& Medicine, 50, 495-509. https://doi.org/10.1016/j.freeradbiomed.2010.11.029

[13] Jonas, C.R., Ziegler, T.R. and Gu, L.H. (2002) Extracellular Thiol/Disulfide Redox State Affects Proliferation Rate in a Human Colon Carcinoma (Caco2) Cell Line. Free Radical Biology \& Medicine, 33, 1499-1506. https://doi.org/10.1016/S0891-5849(02)01081-X

[14] Smeyne, M. and Smeyne, R.J. (2013) Glutathione Metabolism and Parkinson's Disease. Free Radical Biology \& Medicine, 62, 13-25. https://doi.org/10.1016/j.freeradbiomed.2013.05.001

[15] Steele, M.L., Fuller, S. and Maczurek, A.E. (2013) Chronic İnflammation Alters Production and Release of Glutathione and Related Thiols in Human U373 Astroglial Cells. Cellular and Molecular Neurobiology, 33, 19-30. https://doi.org/10.1007/s10571-012-9867-6

[16] Lluis, J.M, Morales, A. and Blasco, C. (2005) Critical Role of Mitochondrial Glutathione in the Survival of Hepatocytes during Hypoxia. The Journal of Biological Chemistry, 280, 3224-3232. https://doi.org/10.1074/jbc.M408244200

[17] Himmelfarb, J., McMonagle, E. and McMenamin, E. (2000) Plasma Protein Thiol Oxidation and Carbonyl Formation in Chronic Renal Failure. Kidney International, 58, 2571-2578. https://doi.org/10.1046/j.1523-1755.2000.00443.x

[18] First, M.B. and Pincus, H.A. (2002) The DSM-IV Text Revision: Rationale and Potential İmpact on Clinical Practice. Psychiatric Services, 53, 288-292.

https://doi.org/10.1176/appi.ps.53.3.288

[19] Kuloglu, M., Atmaca, M., Tezcan, E., Ustundag, B. and Bulut, S. (2003) Antioxidant Enzyme and Malondialdehyde Levels in Patients with Panic Disorder. Neuropsychobiology, 46, 186-189. https://doi.org/10.1159/000067810

[20] Herken, H., Akyol, O., Yilmaz, H.R., Tutkun, H., Savas, H.A. and Ozen, M.E. (2006) Nitric Oxide, Adenosine Deaminase, Xanthine Oxidase and Superoxide Dismutase in Patients with Panic Disorder: Alterations by Antidepressan Treatment. Human Psychopharmacology, 21, 53-59. https://doi.org/10.1002/hup.742 
[21] Biswas, S., Chida, A.S. and Rahman, I. (2006) Redox Modifications of Protein Thiols: Emerging Roles in Cell Signaling. Biochemical Pharmacology, 28, 551-564. https://doi.org/10.1016/j.bcp.2005.10.044

[22] Cremers, C.M. and Jakob, U. (2013) Oxidant Sensing by Reversible Disulfide Bond Formation. The Journal of Biological Chemistry, 288, 26489-26496. https://doi.org/10.1074/jbc.R113.462929

[23] Jones, D.P. and Liang, Y. (2009) Measuring the Poise of Thiol/Disulfide Couples in Vivo. Free Radical Biology \& Medicine, 47, 1329-1338. https://doi.org/10.1016/j.freeradbiomed.2009.08.021

[24] Rodrigues, S.D., Batista, G.B. and Ingberman, M. (2012) Plasma Cysteine/Cystine Reduction Potential Correlateswith Plasma Creatinine Levels in Chronic Kidney Disease. Blood Purification, 34, 231-237. https://doi.org/10.1159/000342627

\section{Submit or recommend next manuscript to SCIRP and we will provide best} service for you:

Accepting pre-submission inquiries through Email, Facebook, LinkedIn, Twitter, etc. A wide selection of journals (inclusive of 9 subjects, more than 200 journals)

Providing 24-hour high-quality service

User-friendly online submission system

Fair and swift peer-review system

Efficient typesetting and proofreading procedure

Display of the result of downloads and visits, as well as the number of cited articles Maximum dissemination of your research work

Submit your manuscript at: http://papersubmission.scirp.org/

Or contact ijcm@scirp.org 